\title{
Pathological characterization of lesions and bacteriological isolation of causative agents of swine tuberculosis at Bishoftu and Addis Ababa Abattoirs, Bishoftu, Ethiopia
}

\begin{abstract}
Background: TB in swine is natural and virtually common in developed countries where swine is farmed. Although this re-emerging disease is very common in developing country as well; there is scanty information on the prevalence of swine tuberculosis in Ethiopia. Therefore, this study was developed to assess the prevalence of swine tuberculosis by using bacteriological and histhopathological techniques.
\end{abstract}

Methods/Principal Findings: A cross sectional study was conducted at Bishoftu and Addis Ababa Abattoirs from September 2017 to May 2018 to estimate abattoir based prevalence of tuberculosis in swine, to isolate Mycobacterium species involved and to characterize the lesions. Five hundred and fifty six (556) swine were examined. Tubercle like granulomatous lesion were detected in 19.6\% (109/556) of different organs of which $12 \%(69 / 556)$ was in lymph nodes, $5.7 \%(32 / 556)$ in the lungs, $1 \%(6 / 556)$ in the liver and $0.36 \%(2 / 556)$ in the spleen. Based on microscopic alteration $4.7 \%(26 / 556)$ of lesions were tuberculous granulomatous type with central necrosis, calcified foci, epitheloid cells admixed with lymphocyte and connective tissue boundries, 3.6\% (20/556) pyogranulomatous and $2.5 \%$ (14/556) non necrotic granulomatous lesion without epitheloid cells and connective tissue capsules. The tuberculous lesions found in lymph node were statistically significant $(\mathrm{P}<$ $0.05)$ than in other organs. The multivariable logistic regression analysis showed that old aged swine were more likely to have characteristic tuberculous lesion $(\mathrm{OR}=3.14,95 \%$ CI, 1.62-6.09) than younger ones. From the tissue cultured, 7.5\% (3/40) yield growth on primary culture media. The observed colony morphology was smooth whitish or yellowish color, sticky, off-white and breaks apart easily and two $(5 \%(2 / 40))$ of these growth were acid fast positive by Zeihl-Neelsen staining technique.

Conclusion/Significance: This study highlights that TB is prevalent in swine and pork can be source of tuberculosis to human when consumed under cooked. Therefore, routine abattoir inspections should be conducted, further molecular and biochemical research to isolate the species of microbacteria is highly recommended to elucidate the type of lesion and its magnitude in different body organs by considering large number of swine.

Keywords: mycobacterium tuberculosis, bacilli, Bishoftu, TB
Volume 4 Issue $6-2019$

\author{
Jirata shiferaw,' Tilaye Demissie,' Kassa \\ Demissie,', Gezahegne Mamo,' Gobena \\ Ameni $^{3}$ \\ 'Faculty of Veterinary Medicine, Addis Ababa University, Ethiopia \\ ${ }^{2}$ Department of Animal Science, College of Agriculture and \\ Natural Resource Sciences, Debre Birhan University, Ethiopia \\ ${ }^{3}$ Aklilu Lemma Institute of Pathobiology, Addis Ababa University, \\ Ethiopia
}

Correspondence: Jirata shiferaw, Faculty of Veterinary Medicine, Addis Ababa University, Bishoftu, Ethiopia,Tel 094910244I, Fax 25 I-I I-4339933,

Emailshiferaw.jirata@yahoo.com,jirata47@yahoo.com

Received: December 20, 2019 | Published: December 31, 2019
Abbreviations: SLN, submandibular lymph node; PLN, parotid lymph node; MLN, mesentric lymph node; RLN, retropharyngeal lymph node; LCR, left cranial; LCA, left caudal; RCR, right cranial; RML, right middle; RCA, right caudal; RAC, right accessory lobes of lungs; LR, liver; SP, spleen, TN, tuberculosis

\section{Introduction}

Tuberculosis (TB) is considered as a re-emerging, infectious granulomatous disease in animals and people caused by acid-fast bacilli of the genus Mycobacterium. Tuberculosis occurs frequently in man, domestic and wild animals. The tubercle bacilli are Mycobacterium tuberculosis, the agent of the disease in primates, $M$. bovis in other mammals and M. avium in birds. Swine are susceptible to all the three types of tubercle bacilli. Swine and dogs are susceptible to both M. bovis and M. tuberculosis. Cats are quite resistant to M. tuberculosis. ${ }^{1}$ Tuberculosis continues to be an important disease both in humans and animals. It causes morbidity, mortality and economic loss worldwide. The occurrence of Mycobacterium bovis disease in humans, domesticated and wild animals confirms the relevance of this zoonosis. ${ }^{2-18}$

Swine are natural hosts for mycobacterial infections including those due to M. bovis. ${ }^{19}$ The most common cause of swine tuberculosis is Mycobacterium avium, but infection with mammalian tubercle bacilli, including M. tuberculosis, M. bovis, and M. africanum occur coincident with infections of cattle, wildlife, and human beings. The finding of severe, diffuse tuberculous lesions strongly suggests that Nebrodi black pigs are susceptible for Mycobacterium spp. and that they might act as a distributor for these microorganisms. ${ }^{3}$ Lesions occurring in swine naturally infected with $M$. bovis and $M$. tuberculosis are indistinguishable. Granulomatous lesions are most often found in the cervical, submandibular, and mesenteric lymph nodes, but in advanced disease lesions may also be found in the liver and spleen. Typically, enlarged nodes contain small, white or yellow, caseous foci, usually without any evidence of calcification. Swine 
with disease due to $M$. tuberculosis may have similar regionalized lesions with human beings. Swine are particularly susceptible to $M$. bovis, which is usually acquired from shared grazing or ingestion of contaminated dairy products. This can cause a rapidly progressive, disseminated disease with caseation and liquefaction of lesions. In industrialized countries most tuberculous lesions in swine are caused by bacteria of the $M$. avium complex (M avium ss hominisuis, M. avium ss avium) and $M$. intracellulare. Lesions are most often observed in lymph nodes associated with gastrointestinal tract. However, it is important to emphasize some swine may develop progressive disease involving the liver, spleen and other organs of the abdominal and thoracic cavities. ${ }^{4,20}$

The lesions in swine infected with $M$. tuberculosis are often associated with lymph nodes of the gastrointestinal tracts. Caseous lesions are most commonly found in the mesenteric or submaxillary lymph nodes; however, microscopic lesions have been observed in the portal and thoracic nodes and in the parenchyma of the lungs. ${ }^{2}$ The De initiative diagnosis to check whether a certain herd is infected or not requires a clear evidence of the agent based on bacteriological culturing, histopathology and molecular methods. ${ }^{21,22}$ The main bootle neck for transmission of TB in many country is from wild species and vice versa. According to Pederson et al., ${ }^{5}$ infection in white-tailed deer (Odocoileus virginianus) has been followed by infection in cattle in some Midwestern states. Infection has also been documented in feral swine (Sus scrofa) on the Hawaiian island of Molokai and in various European countries, but no large-scale survey of antibody exposure to the bacteria has been conducted in feral swine in the US. ${ }^{6}$ A major effort to eradicate tuberculosis in swine and in people markedly reduced the incidence of the types of tuberculosis usually seen in swine and people. The serovars of avian type TB found in confinement-raised swine have been found in people may translate into increased concern for TB in both swine and poultry. Shared research in human and animal health can efficaciously speed the development of new diagnostic tests for humans and livestock, and improve TB surveillance, control, and eradication programs. ${ }^{1,20}$

The main objective of the current study was therefore is the first of its kind in providing baseline information with regard to pathological and histopathological study. The ultimate aim was pathological and bacteriological methods; were used for the diagnosis of swine tuberculosis. Correlation of gross, microscopic and bacteriological findings to increase the sensitivity of TB detection provided the true epidemiological status of the disease and useful information in the swine population in Ethiopia that can be utilized for future research and disease control purpose at national and global level TB control efforts and reducing the risk of its zoonotic significance.

\section{Methods}

\section{Study area and study population}

The study was conducted from September 2017 to May 2018 in Addis Ababa and Bishoftu abattoirs. These two abattoirs were selected purposively because they were the only abattoirs slaughtered swine. This animal species has been brought to the abattoirs from different towns of the country. ${ }^{7}$ The geographical location of Bishoftu is at a latitude and longitude of $8^{\circ} 45^{\prime} \mathrm{N}$ and $38^{\circ} 59^{\prime} \mathrm{E}$, respectively, with an elevation of 1920 meters above sea level. The reliable seasonal rainfall and temperature prediction would have several advantages for record of disease prevalence and increased agricultural productivity in the country. ${ }^{8}$ Apart from Addis Ababa and Bishoftu, swine were brought for slaughter to these abattoirs from surrounding Oromia Zone, Sabata and Burayyu which are at most within $60 \mathrm{~km}$ radii from Addis Ababa. Swine brought from each area were kept under intensive management system, feed poultry manure and left over (disposed carcass of other animals) from abattoirs.

Swine brought to Addis Ababa and Bishoftu abattoirs during the study periods for slaughter were study animals without discrimination of their age, sex, breed and body condition scores. Accordingly, 335 swine, from Addis Ababa and its surrounding and Bishoftu town, brought to Addis Ababa and 221 to Bishoftu abattoirs in total 556 swine were included in the current study. The average number of swine presented to slaughter per day was about 15 to 25 swine in Addis Ababa abattoir and about 5 to 10 swine in Bishoftu abattoir. Slaughtering of swine was done once in a week, commonly, on Monday. (Figure 1).

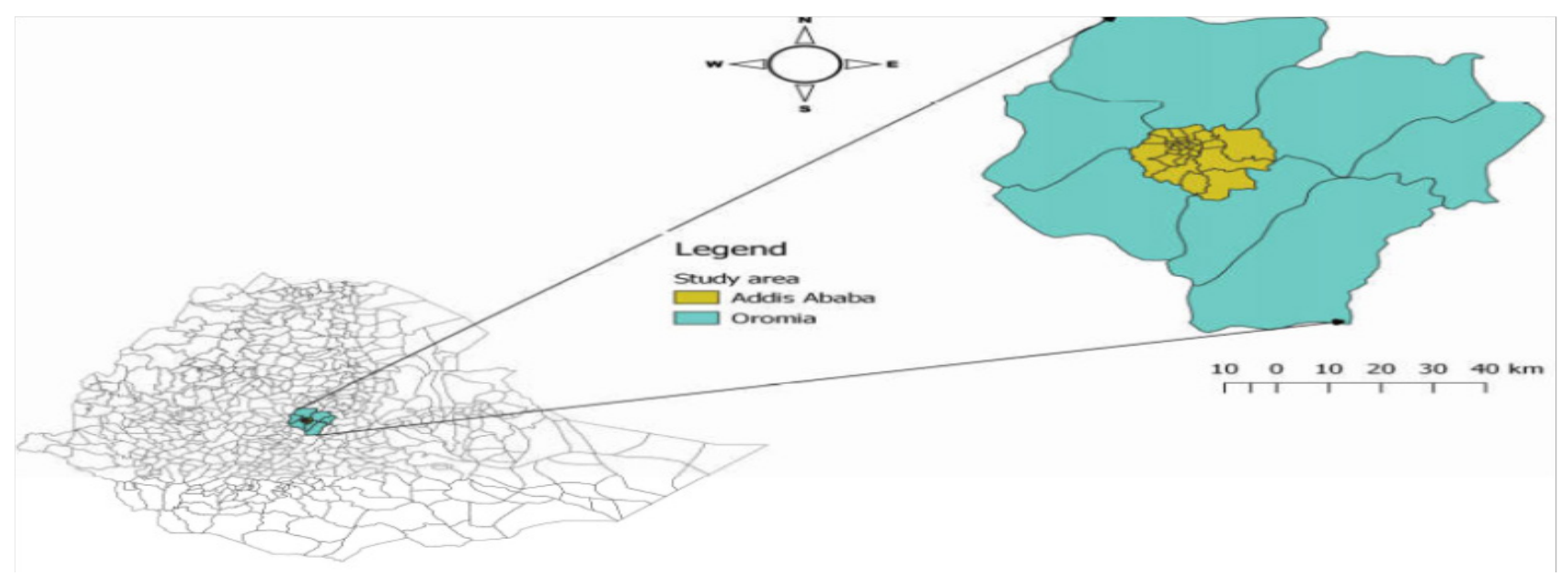

Figure I Map of study area- Addis Ababa and its surrounding Oromia Zone. 


\section{Study design and sample size}

A cross-sectional study was employed for the current study. Previous studies on the abattoir gross lesions prevalence of swine TB were reported to be $3.5 \%(34 / 984)^{9}$ and $5.8 \%(49 / 841)^{10}$ in central Ethiopia. However, there was no a study report based on histopathological diagnostic techniques. Therefore, for the current study, the required sample size were calculated by considering $50 \%$ expected prevalence $(\mathrm{p}), 95 \%$ confidence interval $(\mathrm{z}=1.96)$ and $5 \%$ desired absolute precision (d). The sample size was determined by making use of the formula after Thrusfield and Christley. ${ }^{23}$

$$
\text { Hence, } n=\frac{(z)^{2} p(1-p)}{d^{2}}
$$

Where $\mathrm{n}=$ is the required sample size, $\mathrm{p}=$ is the expected prevalence, $\mathrm{z}=$ multiplier of the $95 \%$ confidence interval and $\mathrm{d}=$ desired absolute precision. By making use of the above formula the calculated sample size (n) was 384 ; however, to increase precision 556 swine were chosen from the two abattoirs.

\section{Sample collection and sample processing}

Non probability sampling method was employed to all slaughtered swine at Addis Ababa and Bishoftu abattoir. Hence, antemortem examination was carried out on all live swine. These included any abnormality in movement (gait), reaction to touch and sound, any visible discharges from natural orifices, coughing, lesions on body and body conditions. Data such as age, sex, origin and body condition scores were collected during antemortem inspection by discussion with the owners and abattoir swine care providers. Postmortem inspections were done and all collected tissues were cut in thin slices (roughly 3 $\mathrm{mm}$ wide), and the presence of macroscopic TBL was recorded ${ }^{11}$ and Di Marco et al., ${ }^{24}$ by observation of any visible gross lesions and by palpation of organs and lymph nodes during and/or after the animals were slaughtered. The lymph nodes inspected include submandibular, mesenteric, retropharyngeal, parotid, mediastinal, tracheobronchial lymph nodes as well as lungs, liver and spleen were inspected.

Pathology scoring was conducted on tissues with tuberculous lesions to determine the severity of the lesions. The lungs and lymph nodes were removed for the investigation of tuberculous lesions based on semi-quantitative procedure developed previously by Ameni et al. ${ }^{12}$ Briefly, lesions in the lobes of the lungs were scored separately as follows: $0=$ no visible lesions; $1=$ no gross lesions but lesions apparent on slicing of the lobe; 2 = fewer than five gross lesions; $3=$ more than five gross lesions; 4 = gross coalescing lesions. The scores for the individual lobe were added to generate lung score. Similarly, the severity of gross lesions in individual lymph node was scored as follows: $0=$ no gross lesions; $1=$ small lesion at one focus; $2=$ small lesions at more than one focus; 3 = extensive necrosis. Scores of individual lymph node were added and generated the lymph node score. The total pathology score per swine was obtained from the sum of lung and lymph node scores.

Gross lesions indicative of TB were observed on lung and/ or lymph nodes to collect tissue samples. Briefly, approximately $2 \mathrm{~mm}$ thick tissue specimens from active lesions were collected into sterile universal bottles filled with $5 \mathrm{ml}$ of $0.9 \%$ saline solution and transported on ice box packed with ice packs to keep the cold chain until they reached Aklilu Lama Institute of Pathobiology (ALIPB) TB laboratory for Mycobacterium culture. Whenever immediate culturing was not done then samples were kept at $4^{\circ} \mathrm{C}$ for two days and refrigerated at $-20^{\circ} \mathrm{C}$ when to stay for longer time to detect mycobacteria. ${ }^{13}$ Mycobacterial culturing was conducted under biocontainment safety cabinet level two (BSC-II) facilitated with HEPA filter (negative pressure). A presumptive diagnosis was made by histopathology by collecting tissue samples of $3-5 \mathrm{~mm}$ in $10 \%$ buffered formalin from lung, liver, spleen and lymph nodes including active part of the lesion and some surrounding apparently normal tissue $^{14}$ and taken to pathology laboratory of the National Animal Health Diagnosis and Investigation Center (NAHDIC).

\section{Histopathology}

Briefly, the formalin fixed tissues were trimmed to $2-3 \mathrm{~mm}$, dehydrated in increasing alcohol concentration, cleared in three passes of xylene and impregnated with molten paraffin. Tissue block were made and then, the tissues were sectioned at $5 \mu \mathrm{m}$ thickness and the sectioned tissue (ribbon) was straighten on water bath. Then, the ribbon was adhered on frost ended and clear slide which is labeled and put in an incubator (annex I). The slides were deparaffinized in 3 changes of xylene, hydrated in decreasing alcohol concentration, placed in hematoxylin, rinsed in tap water, decolorized in acid and checked for differentiation of nucleus and cytoplasm. Then, rinsed again in tap water and stained in Eosin. Dehydrate again in increased alcohol concentration, clear in three changes of xylene and mount cover slide with Canada Balsum. Finally, the slides were read under the microscopic. ${ }^{14}$ Samples from lymph nodes, liver and spleen were categorized according to Varello et al., ${ }^{25}$ as follows: Positive: tubercular granuloma displaying central necrosis with or without mineralization surrounded by macrophages, lymphocytes, plasma cells, neutrophils, epithelioid cells, and Langerhan's giant cells, and enclosed partly or completely by a thin capsule. Inconclusive: lesion characterized by irregular with no capsulated clusters of epithelioid macrophages; associated with a not Langerhan's-type multinucleated giant cells and necrosis. Negative: features not consistent with tubercular granuloma, including significant eosinophilic infiltrates and lymphoid hyperplasia.

\section{Mycobacterial isolation}

The 40 granulomatous samples were collected from lungs and lymph nodes and processed for isolation of mycobacteria in accordance with Ameni et al., ${ }^{15}$ and OIE. ${ }^{14}$ The specimens were sectioned using sterile blades, minced with scissors and homogenized with a sterile mortar and pestle under a biological safety cabinet level two (BSC_II). The homogenates were decontaminated by adding an equal volume of $4 \% \mathrm{NaOH}$ on the sample in order to remove contaminants and $3 \mathrm{ml}$ distilled water or PBS. Then, the processed specimens were centrifuged at 3,000 rpm for 15 minutes to concentrate the mycobacteria. The supernatant was discarded and the sediment was neutralized by $1 \%(0.1 \mathrm{~N}) \mathrm{HCl}$ using phenol red as an indicator. Neutralization was achieved when the color of the solution changed from purple to yellow. ${ }^{14} \mathrm{Next}, 0.1 \mathrm{ml}$ of suspension from each sample was spread onto a slant of Lowenstein Jensen (L-J) medium. Duplicate slants were used in which one was seeded on L-J enriched with sodium pyruvate and the other enriched with glycerol. ${ }^{14}$ The cultures were incubated aerobically at $37^{\circ} \mathrm{C}$ and $5 \% \mathrm{CO}_{2}$ in slant position for one week with daily observation and 8-12 weeks in an upright position with weekly observation for growth of colonies. Positive cultures were confirmed with Ziehl Nelseen staining and preserved with freezing media while at the same time heat killed 
in water bath at $80^{\circ} \mathrm{C}$ for $45-60$ minutes. The frozen and heat killed samples were stored at $-20^{\circ} \mathrm{C}$ for further molecular typing.

\section{Statistical analysis}

The field collected data was entered, classified, filtered and coded using Microsoft Excel Spread Sheet (2010) and analyzed using the software package STATA $13^{26}$ and IBM SPSS version 20. Prevalence was calculated as the proportion of suspected lesion positive animals from the total number of animals sampled. ${ }^{23}$ Descriptive statistics was used for statistical summary. Univariable analysis and Chisquare $(\chi 2)$ test was performed to evaluate the statistical association of different risk factors with the result of gross and histopathology. Multivariable logistic regression analyses were used to analyze strength of association of the potential risk factors after their p-value was identified to be $<0.05$ in univariable analysis. Effects were reported as statistically significant for the p-values less than 0.05 . In cases of estimating the effect of different risk factors in terms of OR

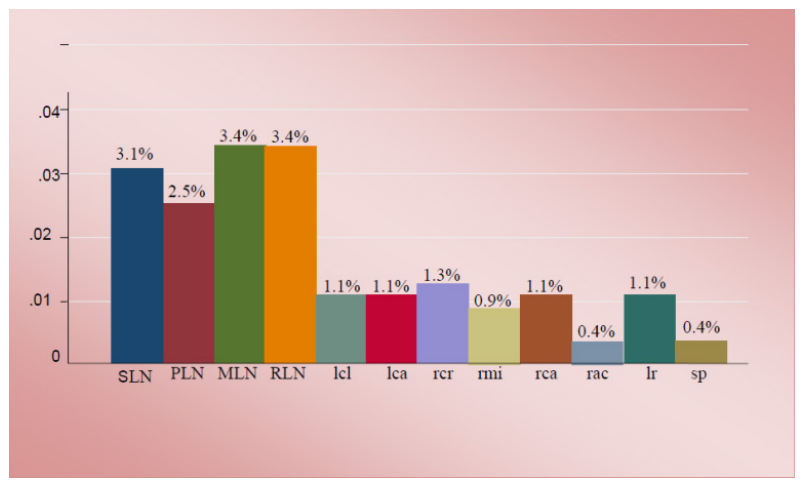

Figure 2 The mean prevalence of tuberculous like lesions in lymph nodes, lungs, liver and spleen.

**SLN, submandibular lymph node; PLN, parotid lymph node; MLN, mesentric lymph node; RLN, retropharyngeal lymph node; LCR, left cranial; LCA, left caudal; RCR, right cranial; RML, right middle; RCA, right caudal; RAC, right accessory lobes of lungs; LR, liver; SP, spleen.

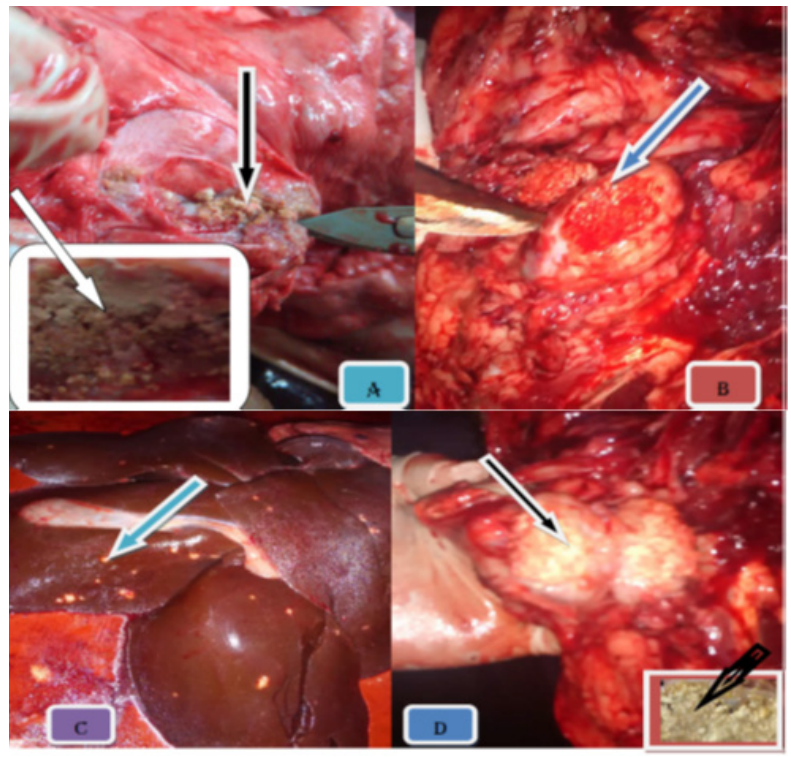

Figure 3 Gross TB like lesion in different organs. Note: The typical caseuos calcified on left caudal lung $(A)$, caseous exudates lesions in retropharyngeal lymph node (B), multifocal hepatic nodules (C), and typical caseous exudates in submandibular lymph node; after parafinized (black arrow) (D). with corresponding $95 \%$ confidence interval, statistical significance was assumed.

\section{Results}

\section{Post-mortem and gross pathology}

On the basis of gross pathology, in 556 slaughtered swine showed that $19.6 \%(109 / 556)$ were found to have tuberculous like lesions in parenchymatous organs of which $12 \%(69 / 556)$ were from lymph nodes, $5.7 \%(32 / 556)$ of the lungs, $1 \%(6 / 556)$ of the liver and $0.36 \%$ $(2 / 556)$ of the spleen (Figure 2). The tuberculous like lesions found in lymph nodes, particularly those of mesenteric and retropharyngeal, were statistically significant $(P<0.05)$ than in lungs and associated lymph node. The majority of the lesions were considered to be of typical tuberculous lesions characterized by central round, oval, or irregular, often coalescing areas of caseous necrosis and calcification. The calcified lesions were exceedingly distributed in different lymph nodes and parts of the lungs (Figure 3).

Pathological lesions were commonly seen in mesenteric lymph node 3.4\% (19/556) and retropharyngeal lymph node 3.4\% (19/556) (Table 1). Among lung lobes found with TB like lesion right cranial, left caudal and left cranial lobes had 5 pathological lesion score with frequency $1.3 \%(7 / 556), 1.1 \%(6 / 556)$ and $1.1 \%(6 / 556)$ respectively (Table 2).

Table I Frequency of lesions in lymph nodes, liver and spleen based on pathological score

\begin{tabular}{llll}
\hline $\begin{array}{l}\text { Lymph } \\
\text { nodes }\end{array}$ & $\begin{array}{l}\text { Total pathological } \\
\text { score }\end{array}$ & Frequency & Percent (\%) \\
\hline SLN & 3 & 17 & 3.1 \\
PLN & 2 & 14 & 2.5 \\
MLN & 4 & 19 & 3.4 \\
RLN & 4 & 19 & 3.4 \\
Lr & - & 6 & 1.1 \\
Sp & - & 2 & 0.4 \\
\hline
\end{tabular}

* SLN, submandibular lymph node; PLN, parotid lymph node; MLN, mesentric lymph node; RLN, retropharyngeal lymph node; LR, liver; SP, spleen

Table 2 Frequency of lesion in different lobes of the lung based on pathological score

\begin{tabular}{llll}
\hline $\begin{array}{l}\text { Lobes of } \\
\text { lung }\end{array}$ & $\begin{array}{l}\text { Total pathological } \\
\text { score }\end{array}$ & Frequency & Percent (\%) \\
\hline Lcr & 5 & 6 & 1.1 \\
Lca & 5 & 6 & 1.1 \\
Rcr & 4 & 7 & 1.3 \\
Rml & 4 & 5 & 0.9 \\
Rca & 5 & 6 & 1.1 \\
Rac & 4 & 2 & 0.4 \\
\hline
\end{tabular}

*LCR, left cranial; LCA, left caudal; RCR, right cranial; RML, right middle; RCA, right caudal; RAC, right accessory lobes of lungs

Based on the stages of granuloma, 4.7\% (26/556) lesions were calcified granulomas, $4.1 \%$ (23/556) lesions were necrotized, 3.6\% $(20 / 556)$ lesions were pyogranulomatous and $2.5 \%(14 / 556)$ lesions were resulted granulomas without calcification (Table 3 ). 
Table 3 Frequency of different types of lesions in lymph nodes, lungs, liver and spleen

\begin{tabular}{lll}
\hline Types of lesions & Frequency & Percent (\%) \\
\hline CG & 26 & 4.7 \\
N & 23 & 4.1 \\
PG & 20 & 3.6 \\
G & 14 & 2.5
\end{tabular}

\section{Association of different risk factors considered with gross lesions}

The univariable and multivariable logistic regression analyses of the different putative risk factors considered for tuberculous like lesion positivity were indicated in Tables $4 \& 5$ respectively. Swine with poor body condition had higher odd (likelihood) to develop TB like lesions $(\mathrm{OR}=0.21,95 \% \mathrm{CI}, 0.11-0.38)$ than good body conditioned swine. Old aged swine were positive to have tuberculous like lesions $(\mathrm{OR}=3.14,95 \% \mathrm{CI}, 1.62-6.09)$ than younger ones.

*Note: CF, calcified granuloma; G, granulomas without calsification; N, necrotized lesions; PG, pyogranulomatous

Table 4 Univariable logistic regression of different risk factors to postmortem lesion findings

\begin{tabular}{|c|c|c|c|c|c|c|}
\hline Variables & No. Swine & No. Positive & Prevalence of TB (\%) & OR & $\chi^{2}$ & P-value \\
\hline \multicolumn{7}{|l|}{ Sex } \\
\hline Female & 297 & 43 & 14.47 & & 0.1 & 0.75 \\
\hline Male & 259 & 40 & 15.44 & 1.07 & & \\
\hline \multicolumn{7}{|l|}{ BCS } \\
\hline Poor & 264 & 21 & 7.9 & & 26.81 & $0.000 *$ \\
\hline Moderate & 174 & 28 & 16.09 & 0.47 & & $0.010 *$ \\
\hline Good & 118 & 34 & 28.81 & 0.21 & & $0.000 *$ \\
\hline \multicolumn{7}{|c|}{ Age (in year) } \\
\hline I & 254 & 26 & 10.23 & & 27.58 & \\
\hline 2 & 212 & 28 & 13.2 & 1.33 & & 0.319 \\
\hline 3 & 72 & 19 & 26.38 & 3.14 & & $0.001 *$ \\
\hline 4 & 18 & 10 & 55.56 & 10.96 & & $0.000 *$ \\
\hline
\end{tabular}

BSC, body condition score; $\chi 2$, Chi-square; OR, odds ratio(crude); $*$ = statistically significant

Table 5 Multivariate analysis of risk factors associated with postmortem lesion findings

\begin{tabular}{lllllll}
\hline Variables & No. Swine & $\chi 2$ & OR & \multicolumn{2}{c}{$95 \%$ Cl for OR } & P-value \\
\hline BCS & & 26.81 & & Lower & Upper & $0.000^{*}$ \\
Poor & 264 & & & & & \\
Medium & 174 & & 0.47 & 0.26 & 0.83 & \\
Good & 118 & & 0.21 & 0.11 & 0.38 & \\
Age (in year) & & & & & & $0.000^{*}$ \\
I & 27.58 & & & & \\
2 & 212 & & 1 & & & \\
3 & 72 & & 1.3 & 0.75 & 2.23 & \\
4 & 18 & 3.14 & 1.62 & 6.09 & \\
& & & 10.95 & 3.97 & 30.22 &
\end{tabular}

$\mathrm{BSC}=$ body condition score; $\mathrm{Cl}=$ Confidence interval; $\chi 2=$ Chi-square; $\mathrm{OR}=$ odds ratio (adjusted); $*$ statistically significant

\section{Histopathological lesions}

The most characteristic microscopic lesion was tubercle granuloma with central necrosis and calcification. The central area is made of necrotic cellular debris, calcium deposits, and connective tissue capsule walled off the granuloma from the surrounding tissue.
The next layers from center to outer were made up of lymphycytes, macrophages, epitheloid macrophages distributed under connective tissue layers (Figure 4A). The frequency of characteristic tubercle granuloma was $4.7 \%(26 / 556)$ (Figure 4B). The presence of concomitant pyogranulomatous and granulomatous lesions in 
different organs was observed in $7.1 \%(40 / 556)$. Some granulomas were characterized by necrotic foci and intense calcification and fibrosis with absence of epithelioid cells. Multiple small granulomas in the lymph node (Figure 5) with less dense lymphocyte at periphery and epitheloid cells surrounding the deep outer lymphatic layer of the granuloma.

Multifocal hepatonecrosis with total removal of hepatocytes and infiltration of infilammatory cells (mixed mononuclear and polymorphoneuclear leukocytes) (Figure 6). At the periphery of this central necrosis are huge infiltrations of inflammatory cells (eosinophils, neutrophils and lymphocytes) in the portal triad region. Some foci of hepatic degeneration with swollen hepatocytes.

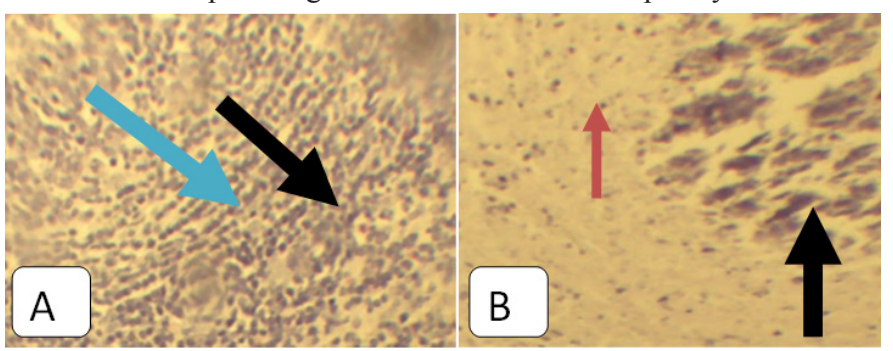

Figure 4 A) Characteristic epitheloid macrophages in the lymph nodes. Note; The epitheloid macrophages shown as elongated (Blue arrow) and the lymphocytes in the next layers (black arrows). H \& E stains (I0x). B) The characteristic granulomas in the lymph node with calcium deposits (red arrow) at the center, followed by sever necrosis and cellular debris (black arrow) immediate to calcium deposits. $\mathrm{H} \& \mathrm{E}$ stain (I0x).

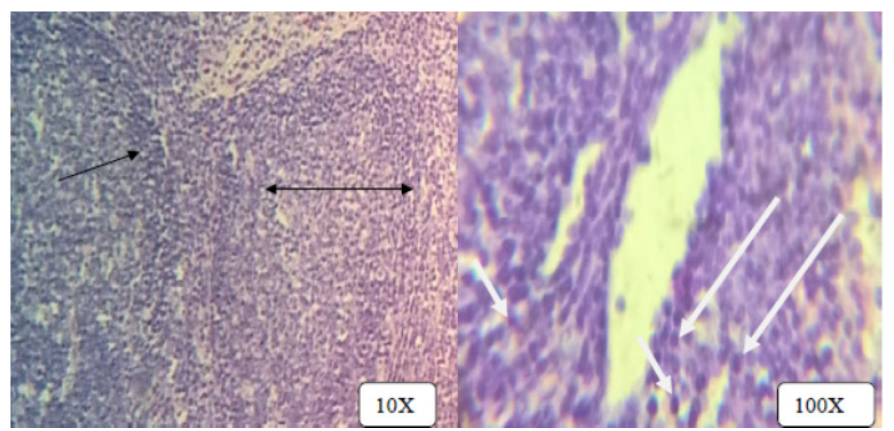

Figure 5 Multiple small granulomas in the lymph node with central necrosis, layer of dense lymphocyte at periphery (double headed black arrows) admixed with epitheloid cells (white arrow). H \& E stains.

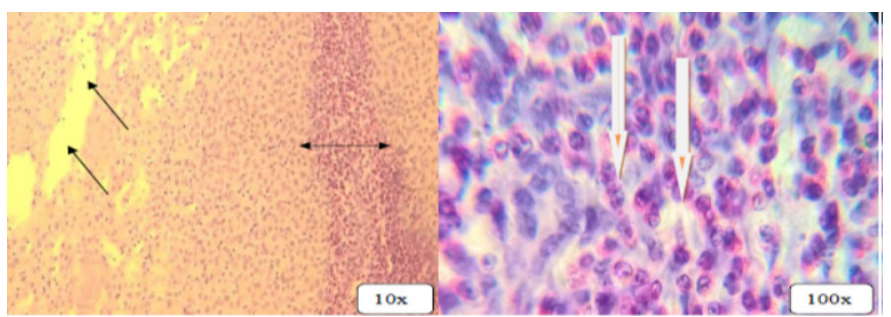

Figure 6 Multifocal hepatonecrosis with total removal of hepatocytes (black arrows), infiltration of inflammatory cells into portal traiads (double head arrows) and infiltration of inflammatory cells (white arrows). H \& E stains.

\section{Mycobacterial isolation and characterization}

Mycobacterial culturing was conducted from characteristic tubercle lesions of which $7.5 \%$ (3/40) showed growth on primary culture media. Of those cultures which showed visible colony, $5 \%$ $(2 / 40)$ were seen on Lowenstain-Jenson (L-J) media enriched with glycerol and the rest $2.5 \%(1 / 40)$ were grown on L-J media enriched with pyruvate. Three grown cultures were subjected to Zeihl-Neelsen staining technique in order to check the presence of acid fast bacilli. $5 \%(2 / 40)$ of the cultures were found to be acid fast positive. The observed colonial morphology was smooth whitish or yellowish color, sticky, off-white and breaks apart easily (Figure 7). The results attained of Z-N staining were cocci, short and some long rod shaped and also found in single and clumps (Figure 8), (Table 6).

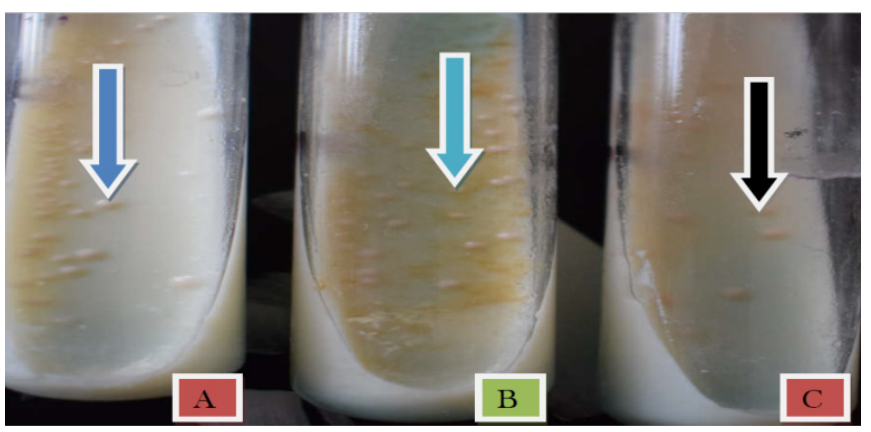

Figure 7 Grown bacilli on L-J culture media. Note: (A \& B) Colonies growth on L-J medium supplemented with glycerol; (C) Colony growth on L-J medium supplemented with pyruvate (Arrows indicate colony growth which is characterized as smooth, whitish or yellowish in color).
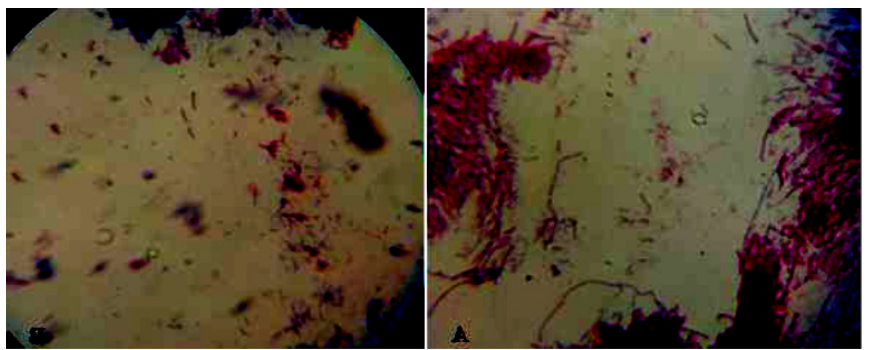

Figure 8 AFB staining positive isolates from mycobacteriological culture colony grows. From lymph nodes (Mesentric and retropharyneal) TB lesion (arrows indicate acid fast bacilli which a short and long, in Single or clump).

\section{Discussions}

There is little information on $\mathrm{TB}$ in swine and no report on histopathologic diagnosis with few published on swine TB in Ethiopia. The recognition of lesions of swine tuberculosis during meat inspection is of paramount importance for the surveillance and control of TB infection. Domingo et al., ${ }^{27}$ indicated that failure to detect a lesion during abattoir meat inspection has the greatest significance in cattle with single lesion. As the same time; once the lesion is missed there is no further chance of detecting the disease in the animal. This idea also supports the chance of missing small sized lesions while inspecting swine carcasses at abattoir. Furthermore, the early slaughtering of swine before development of tuberculosis lesions in different organs and inadequate illumination in an inspection room of the abattoirs are the major factors determining the detection of tuberculous lesion/s. The slaughtered swine at an early age without sufficient time for lesion development and progression in the present study $(45.6 \%(254 / 556))$, was in agreement with the findings of the Domingo et al. ${ }^{27}$

The present study revealed tuberculous like lesions in $19.6 \%$ $(\mathrm{n}=109)$ of swine carcasses inspected which was comparable to $19.8 \%$ reported by Cleaveland et al. ${ }^{28}$ in cattle slaughtered in rural Tanzania. The current result was higher than $5.8 \%$ reported by 
Arega et al. ${ }^{10}$ in Addis Ababa and Bishoftu abattoir in swine, 7.96\% reported by Regassa ${ }^{29}$ in Wolaita abattoir in cattle, $8.8 \%$ reported by Biffa et al., ${ }^{30}$ in Hawassa municipal abattoir in cattle, 9\% reported by Nemomsa et al., ${ }^{16}$ in Butajira abattoir in cattle and $11.50 \%$ reported by Abdurohaman $^{31}$ in Butajira abattoir in cattle. On the other hand, the finding of this study was lower than $24.7 \%$ reported by Biffa et al., ${ }^{30}$ and $24 \%$ by $\mathrm{Mamo}^{32}$ in cattle at Adama municipal abattoir. The higher prevalence recorded in the present study could be due to the fact that swine feed mainly poultry meal and their offal's. Furthermore, the variations in prevalence could be attributed to the possible differences in the epidemiology of the disease in different species of animals, origin, housing, age, body condition scores of the animals and types of production system. The majority of swine under this study were originated from intensive poor management production system. According to Ameni et al., ${ }^{15}$ Radostits et al., ${ }^{42}$ and Mamo et al., ${ }^{17}$ the intensive livestock management system could contribute to the development of mycobacterial infections than the extensive livestock management system which is in line with the current finding.

Table 6 Bacterial culture result from tissues suspected tuberculosis lesions

\begin{tabular}{|c|c|c|c|c|c|}
\hline \multicolumn{5}{|l|}{ Growth } & \multirow{3}{*}{ Z-N stain Positive (\%) } \\
\hline \multirow[t]{2}{*}{ Sample type } & \multicolumn{2}{|c|}{ L-J media with pyruvate } & \multicolumn{2}{|c|}{ L-J media with glycerol } & \\
\hline & Total & Positive & Total & Positive & \\
\hline SLN & 10 & $\mathrm{I}(2.5)$ & 10 & - & - \\
\hline PLN & 7 & - & 7 & - & - \\
\hline MLN & 10 & - & 10 & $\mathrm{I}(2.5)$ & $I(2.5)$ \\
\hline RLN & 8 & - & 8 & - & - \\
\hline Lung & 5 & - & 5 & $\mathrm{I}(2.5)$ & $\mathrm{I}(2.5)$ \\
\hline Total & 40 & $\mathrm{I}(2.5)$ & 40 & $2(5)$ & $2(5)$ \\
\hline
\end{tabular}

*SLN, sub-mandibular lymph node; PLN, parotid lymph node; MLN, mesenteric lymph node; RLN, retropharyngeal lymph node

The difference in the prevalence of gross lesions between sexes was statistically insignificant $\left(\chi^{2}=0.1\right.$ and $\left.P>0.05\right)$. The overall gross lesion prevalence in different organs of the both sex was $19.6 \%$ (109/556). This study result is higher than the previous studies by Teklu et al., ${ }^{33}$ who reported $4.53 \%$ in cattle in Hossana, Shitaye et al., ${ }^{9}$ who reported 3.5\% in swine in Addis Ababa, Arega et al., ${ }^{10}$ who reported 5.8\% in swine in Addis Ababa, and to some extent consistent with the report by Cleaveland et al. ${ }^{28}$ who reported $19.8 \%$ in cattle in Tanzania. The possible reason for this discrepancy might be due to the difference in lesions distribution of female and male animals, in which it was higher in females, sample size and study methodology. Less number of male swine and relatively more number of adult female swine as a means of culling from the breeding stock were presented for slaughter to the study abattoirs during the current study duration.

There was statistically significant difference in this study $\left(\chi^{2}=\right.$ 27.58 and $\mathrm{P}<0.05$ ) between age and lesions in different organs (lungs, lymph node, spleen and liver). This result is in consistent with the reports of Gebremedhin et al., ${ }^{34}$ who reported 2.6\% in Dilla Municipal Abattoir in cattle and Nemomsa et al., ${ }^{16}$ who reported $9 \%(p<0.05)$ in Butajira abattoir in cattle. The older swine $(\mathrm{OR}=10.95)$ were nearly eleven times positive to have the gross pathological lesions than the younger ones. The results of the current finding also agreed with the findings of Barwinnek and Taylor, ${ }^{35}$ Ameni et al., ${ }^{15}$ Regassa et al. ${ }^{36}$ and Biffa et al., ${ }^{37}$ in cattle who indicated as the age of the animal increases the probability of acquiring TB infection also increases. O'Reilly et al. ${ }^{38}$ pointed out that; the protective capability is declining in aging animals due to a weaker immune system. Furthermore, Humblet et al. ${ }^{39}$ indicated stresses, malnutrition and immunosuppression increase with age. The findings of all these authors supported the current result. The possible reason of difference between ages in current study will be the number of animals considered in each category is not proportional.
The effect of difference in the prevalence of swine TB among swine having different body condition scores was statistically significant $\left(\chi^{2}=26.81 ; \mathrm{P}<0.05\right)$. The prevalence was highest in swine with poor body condition (47\%) as compared to swine with medium body condition $(21 \%)$ and good ( $8 \%$ ) body conditions. This finding is in agreement with study reported by Nemomsa et al., ${ }^{16}$ who reported the higher prevalence of tuberculosis lesions in poor body condition than good body condition animals $\left(\chi^{2}=10.38\right.$; $\left.\mathrm{p}<0.05\right)$ in Butajira abattoir in cattle. This could be due to the weak protective immune response in poor body conditioned swine when compared to swine with good body condition. Moreover, TB in poor body conditioned swine may result in extensive lesions and wasting of the body due to its chronic nature. The present result is in consistent with previous reports of Collins et al., ${ }^{40}$ Radostits et al., ${ }^{41}$ and Radostits et al., ${ }^{42}$ in those animals with good body condition have relatively good immunological response to the infectious agent than animals with medium and poor body condition scores. On the other hand, the animals with poor body conditions and in nutritional deficiency have reduced resistance to TB which is also in consistence with the report by Doherty et al. ${ }^{43}$

A significant difference between the lungs and lymph node ( $\mathrm{P}$ $<0.05$ ) with lesions was associated with the route of infection. In addition, observation of tuberculous lesions in 3.4\% (19/556) of the mesenteric and retropharyngeal lymph nodes may also imply that, the occurrence of intestinal tuberculosis was found to be more frequent than respiratory form. This finding is in agreement with the report described by Shitaye et al., ${ }^{9}$ who observed tuberculous lesions in $33 \%(5 / 15)$ in mesenteric lymph node of swine that dictated that the occurrence of intestinal tuberculosis is more frequent than respiratory tubercolosis. However, the current finding is in disagreement with Corner [44], Neill et al., ${ }^{45}$ Collins, ${ }^{46}$ Whipple et al., ${ }^{47}$ and Teklu et 
al. ${ }^{33}$ who reported that greater than $84 \%$ TB lesions due to $M$. bovis occurred in the respiratory system indicating inhalation/aerosolization in cattle to be the predominant route of transmission. Thoen et al., ${ }^{1}$ described on the similar that ingestion of contaminated poultry faeces and offals and shared grazing with infected animals and/or ingestion of contaminated dairy products are the major sources of TB infection in swine.

Granuloma with calcification was the lesion most frequently detected in the examined organs and occurred in $4.7 \%$ of the cases (Figure 5) in the current histopathological finding. In consistence to this finding, Shitaye et al., ${ }^{9}$ reported $3.4 \%$ granulomatous lesions typical to swine TB. Granulomatous lesions typical to swine tuberculosis, manifesting granulomas with central necrosis surrounded by epitheloid cells distributed under connective tissue layers, were observed in $7.1 \%$. Consistent to this finding, Whipple et al., ${ }^{47}$ described that manifestation of typical granulomatous lesions in tissues with gross lesions was evident histologically.

The growth rate of mycobacteria on culture media in the current study was $7.5 \%(3 / 40)$. Amanfu ${ }^{48}$ and Cleaveland et al., ${ }^{28}$ pointed out the poor growth rate of $M$. bovis on standard L-J medium. However, Teklu et al., ${ }^{33}$ explained M. avium and M. tuberculosis to be well grown on standard L-J medium. Furthermore, Teklu et al., ${ }^{33}$ indicated the presence of caseous and/or calcified lesions may not be truly tuberculous lesions; Pritchard ${ }^{49}$ and Diguimbaye Djaibe et al., ${ }^{50}$ also implied that viable mycobacteria may not be present in calcified lesions. The $7.5 \%$ culture positivity from the lesion current finding, was lower than $47 \%$ reported by Ameni et al., ${ }^{51} 35 \%$ reported by Müller et al., ${ }^{52} 32 \%$ reported by Shimeles, ${ }^{53} 31.4 \%$ reported by Woyessa et al. ${ }^{54}$ and $23.6 \%$ reported by Araujo et al. ${ }^{55}$ in slaughtered cattle, and $30.6 \%$ by Arega et al., ${ }^{10}$ in swine. However, the present finding was higher than that of Aknaw et al., ${ }^{56}$ who reported $2.9 \%$ in slaughtered cattle at Bishoftu abattoir. This findings justify that the types of culture media and weakly developed and/or trace number of tubercle bacilli in swine tissue may result in lower growth rate.

Mycobacteria in this study were detected by Z-N staining in culture positive samples. This finding was consistent with a previous report by Gracey. ${ }^{57} M$. bovis are often low in bovine specimens and they can be visualized by Z-N only if a limited quantity (at least $5 \times 10^{4}$ mycobacteria/ml) of mycobacteria are present. ${ }^{58}$

\section{Conclusion}

In conclusion, the results of this study show that, histopathological analysis followed by microbacterial isolation and Z-N staining indicated that swine can serve as a source of infection of tuberculosis to swine, human and/or other animal species. However, comparison of cultural isolation of Mycobacterium to pathological lesions indicated culture positivity to be smaller than lesions. This is due to the fact that lesions may be caused by other infectious agents rather than Mycobacterium species. Therefore, the diagnosis of mycobacteriosis in swine on a herd basis is important and usually directed to detect lymph nodes on the first hand and lungs as the second option from swine at slaughter. These results should be considered to prevent misdiagnosis of TB based on gross lesions and to establish specific control measures against these pathogens in pigs and related domestic animal species.

\section{Author contributions}

Conceived and designed the experiments: JS, KD, TD. Performed the experiments: JS, KD. Analyzed the data: KD, TD, SL. Contributed reagents/ materials/analysis tools: GM, GA. Wrote the paper: JS, KD, TD. Revised the final version of the paper for publication: TD, KD, GM, JS.

\section{Acknowledgments}

The authors are grateful to Addis Ababa Abattoir Enterprise and Bishoftu Abattoir for their support during sample collection. We also thankful for TB laboratory staffs at Aklilu Lemma Institute of Pathobiology and Faculty of Veterinary Medicine of Addis Ababa University for their support during the laboratory work.

\section{Ethics statement}

Ethical approval was obtained from Addis Ababa University Colege of Veterinary Medicine dated 14/11/2107 (VM/ERC/05/10/018, 03/01/2018), Bishoftu, Ethiopia.

\section{Conflicts of interest}

The author declares there are no conflicts of interest.

\section{References}

1. Thoen CO, Kaplan B, Thoen TC, et al. Zoonotic Tuberculosis . A Comprehensive One Health Approach Pathogenesis. Medicina (B Aires). 2016;76(3):159-165.

2. Thoen CO, Lobue PA, Enarson DA, et al. Tuberculosis : a re $\square$ emerging disease in animals and humans. Vet Ital. 2009;45(1):135-181.

3. Amato B, Maria T, Biasibetti E, et al. Pathology and genetic findings in a rare case of Mycobacterium caprae infection in a sow. Vet Microbiol. 2017;205:71-74.

4. Thoen CO. Tuberculosis and other Mycobacterial Infections. Swine health. 2013;1-4.

5. Pedersen K, Miller RS, Anderson TD, et al. Limited Antibody Evidence oF Exposure To Mycobacterium Bovis In Feral Swine ( Sus Scrofa ) In The USA. J Wildl Dis. 2017;53(1):30-36.

6. Kerri P, Miller RS, Anderson TD, et al. Limited Antibody Evidence oF Exposure To Mycobacterium Bovis In Feral Swine ( Sus Scrofa ) In The USA. J Wildl Dis. 2017;53(1):30-36

7. CSA (Central Statistical Authority). Federal democratic republic of ethiopia. 2018.

8. Kassa Fekadu. Ethiopian Seasonal Rainfall Variability and Prediction Using Canonical Correlation Analysis (CCA). Earth Sciences. 2015;4(3):112-119.

9. Shitaye JE, Getahun B, Alemayehu T, et al. A prevalence study of bovine tuberculosis by using abattoir meat inspection and tuberculin skin testing data, histopathological and IS 6110 PCR examination of tissues with tuberculous lesions in cattle in Ethiopia. Vet Med (Praha). 2006;51(11):512-522.

10. Arega SM, Conraths FJ, Ameni G. Prevalence of tuberculosis in pigs slaughtered at two abattoirs in Ethiopia and molecular characterization of Mycobacterium tuberculosis isolated from tuberculous-like lesions in pigs. BMC Vet Res. 2013;9:97.

11. Santos N, Geraldes M, Afonso A, et al. Diagnosis of Tuberculosis in the Wild Boar ( Sus scrofa ): A Comparison of Methods Applicable to Hunter-Harvested Animals. PLOS ONE.2010;5(9):12663.

12. Ameni G, Aseffa A, Engers H, et al. Cattle Husbandry in Ethiopia Is a Predominant Factor Affecting the Pathology of Bovine Tuberculosis and Gamma Interferon Responses to Mycobacterial Antigens. Clin VACCINE Immunol. 2006;13(9):1030-1036. 
13. Analysis of Bovine Tuberculosis Surveillance in Accredited Free States. 2009.

14. OIE (office of International des Epizootics). Bovine Tuberculosis. 2006;1-6.

15. Ameni G, Aseffa A, Engers H, et al. High Prevalence and Increased Severity of Pathology of Bovine Tuberculosis in Holsteins Compared to Zebu Breeds under Field Cattle Husbandry in Central Ethiopia. Clin VACCINE Immunol. 2007;14(10):1356-1361.

16. Nemomsa B, Gebrezgabiher G, Birhanu T, et al. Epidemiology of bovine tuberculosis in Butajira, Southern Ethiopia : A cross-sectional abattoir-based study. African J Microbiol Res. 2014;8(33):3112-3117.

17. Mamo G, Abebe F, Worku Y, et al. Bovine tuberculosis and its associated risk factors in pastoral and agro-pastoral cattle herds of Afar Region, Northeast Ethiopia. J Vet Med Anim Heal. 2013;5(6):171-179.

18. Mohamed AM, Abou El Ella GA, Nasr EA. Phenotypic and molecular typing of tuberculous and nontuberculous Mycobacterium species from slaughtered swine in Egypt. J Vet Diagn Invest. 2009;21(1):48-52.

19. De Lisle GW. Mycobacterial infections in swine. Surveillance. 1994;21:23-25

20. Charles O Thoen. Overview of Tuberculosis and other Mycobacterial Infections. 2017.

21. Thoen CO, P Lo Bue, DA Enarson, et al. Tuberculosis: a re-emerging disease of animals and humans. Vet Ital. 2009;45(1):135-181.

22. Medeiros Ldos S, Marassi CD, Figueiredo EE, et al. Potential application of new diagnostic methods for controlling bovine tuberculosis. Braz J Microbiol. 2010;41(3):531-541.

23. Thrusfield M, Christley R. Veterinary Epidemiology. 4th edn. 2018.

24. Di Marco V, Mazzone P, Capucchio MT, et al. Epidemiologica significance of the domestic black pig (Sus scrofa) in maintenance of bovine tuberculosis in Sicily. J Clin Microbiol. 2012;50(4):1209-1218.

25. Varello K, Pezzolato M, Mascarino D, et al. Comparison of histologic techniques for the diagnosis of bovine tuberculosis in the framework of eradication programs. $J$ Vet Diagn Invest. 2008;20(2):164-169.

26. StataCorp. Stata Statistical Software: Release 13. College Station, TX: StataCorp LP. 2013.

27. Domingo M, Vidal E, Marco A. Pathology of bovine tuberculosis. Res Vet Sci. 2014;(97 Suppl):S20-S29.

28. Cleaveland S, Shaw DJ, Mfinanga SG, et al. Mycobacterium bovis in rural Tanzania: risk factors for infection in human and cattle populations. Tuberculosis. 2007;87(1):30-43.

29. Regassa A. Preliminary study on bovine tuberculosis in Wolaita-Sodo, South Ethiopia. DVM Thesis. Faculty of Veterinary Medicine, Addis Ababa University, Debre-Zeit, Ethiopia. 1999.

30. Biffa D, Inangolet F, Oloya J, et al. Prevalence of bovine tuberculosis in Ethiopian slaughter cattle based on post-mortem examination. Trop Anim Health Pro. 2009;41(5):755-765.

31. Abdurohaman M. Cross sectional study of bovine tuberculosis in Butajira municipal abattoir, South West Ethiopia. J Global Vet. 2009;6:172-179.

32. Mamo B. Efficiency of abattoir inspection to detect tuberculosis lesions in cattle slaughtered at Adama municipality abattoir, central Ethiopia, DVM thesis, Addis Ababa, Ethiopia. 2007;8-15.

33. Teklu A, Asseged B, Yimer E, et al. Tuberculous lesions not detected by routine abattoir inspection: the experience of the Hossana municipal abattoir, Southern Ethiopia. Rev Sci Tech. 2004;23(3):957-964.
34. Gebremedhin G, Gebremedhin R, Gobena A. Prevalence Study of Bovine Tuberculosis and Genus Typing of its Causative Agents in Cattle Slaughtered at Dilla Municipal Abattoir, Southern Ethiopia. African Journal of Basic \& Applied Sciences. 2014;6(4):103-109.

35. Barwinnek F, Taylor NM. Assessment of the socio-economic importance of bovine tuberculosis in Turkey and possible strategies for control or eradication. Turkish-German Animal Health Information Project. General directorate of protection and control, Ankara. Eschborn: Deutsche Gesellschaft fur Technische Zusammenarbeit, 1996;3-45.

36. Regassa A, Tassew A, Amenu K, et al. Across sectional study on bovine tuberculosis in Hawassa town and its surroundings, Southern Ethiopia, TAHP. 2010;42(5):915-920.

37. Biffa D, Inangolet F, Bogale A, et al. Risk factors associated with prevalence of tuberculosis-like lesions and associated mycobacteria in cattle slaughtered at public and export abattoirs in Ethiopia. Trop Anim Health Pro. 2011;43(2):529-538.

38. O'Reilly LM, Daborn CJ. The epidemiology of Mycobacterium bovis infections in animals and man: a review. Tubercle Lung Dis. 1995;76(Suppl 1):1-46.

39. Humblet MF, ML Boschiroli, C Saegerman. Classification of worldwide bovine risk factors in cattle: a stratified approach. Vet Res. 2009;40(5):50

40. Collins CH, Grange JM. The bovine tubercule bacillus. J Appl.Bacteriol. 1994;55(1):13-29.

41. Radostits OM, Blood DC, Gay CC. Diseases caused by Mycobacterium. In: veterinary medicine, a text book of disease of cattle, sheep, swine, goats and horses. 8th edn. London, Bailliere tindu. 1994;748-785.

42. Radostits OM, Gay CC, Hinchelift KW, et al. Veterinary Medicine. A text book of the disease of cattle, sheep, pig, goat and horses. 10th edn. 2007; 1007-1040.

43. Doherty M, Basset H, Quinn P, et al. A sequential study of the bovine tuberculin reaction. Immunology. 1996;87(1):9-14.

44. Corner LA. Post mortem diagnosis of Mycobacterium bovis infection in cattle. Vet Microbiol. 1994;40(1-2):53-63.

45. Neill SD, Pollock JM, Bryson DB, et al. Pathogenesis of $M$. bovis infections in cattle. Vete Mic. 1994;40(1-2):41-52.

46. Collins JD. Factors relevant to $M$. bovis eradication. Irsh Vet J. 1996;49:241-243.

47. Whipple LD, Boline AC, Miller MJ. Distribution of lesion in cattle infected with mycobacterium bovis. J Vet Diagn Invest. 1996;8(3):351354

48. Amanfu W. The situation of tuberculosis and tuberculosis in animal of economic interest. Tuberculosis. 2006;86(3-4):330-335.

49. Pritchard DG. A century of bovine tuberculosis, 1888-1988: conquest and controversy. J Comp Pathol. 1988;99(4):357-387.

50. Diguimbaye Djaibe C, Vincent V, Schelling E, et al. Species identification of non-tuberculous Mycobacteria from humans and cattle of Chad. Schweiz Arch Tierheilkd. 2006;148(5):251-256.

51. Ameni G, Bekele S, Tolosa T. Preliminary study on the impact of Bovine Tuberculosis on the reproductive efficiency and productivity of Holstein dairy cows in central Ethiopia. B. of Ani H and Prn in Afr. 2010;58(3):223-228.

52. Müller B, Dürr S, Alonso, S, et al. Zoonotic Mycobacterium bovis induced Tuberculosis in Humans. Eme Infec Dis. 2008;19(6):899-908.

53. Shimeles SB. Bovine tuberculosis. Epidemiologic aspects and public health implications in and around Debre Birihan. MSc thesis. Ethiopia. 2008 . 
54. Woyessa M, Jibril Y, Ameni G, et al. Molecular Epidemiology of Mycobacterium Tuberculosis Complex at Nekemte Municipality Abattoir, Western Ethiopia. Sci Tech Arts Res. 2014;3(2):167-173.

55. Araújo CP, Osório AAR, Jorge $\mathrm{K}$, et al. Direct detection of Mycobacterium tuberculosis complex in bovine and bubaline tissues through nested- PCR. Brazilian J Micro. 2014;45(2):633-640.

56. Aknaw W. Bovine tuberculosis lesion description with molecular characterization of Mycobacterium species from cattle slaughtered at ELFORA and municipal abattoir, Bishoftu, Ethiopia. Msc In Tropical Veterinary Pathology. 2016
57. Gracey JF. Meat hygiene. 8th edn. Bailliere-Tindall.1986;350-360.

58. Quinn PJ, Carter ME, Markey B, et al. Mycobacterium species. In: clinical veterinary microbiology. London Philadelphia. 1999;157-170. 